\title{
EchoGéo
}

30 | 2014

Varia

\section{La pauvreté urbaine à Mexico : une approche de la diversité des colonies populaires}

\section{Antonine Ribardière and Jean-François Valette}

\section{(2) OpenEdition \\ 12 Journals}

Electronic version

URL: https://journals.openedition.org/echogeo/14020

DOI: 10.4000/echogeo.14020

ISSN: 1963-1197

\section{Publisher}

Pôle de recherche pour l'organisation et la diffusion de l'information géographique (CNRS UMR 8586)

\section{Electronic reference}

Antonine Ribardière and Jean-François Valette, "La pauvreté urbaine à Mexico : une approche de la diversité des colonies populaires", EchoGéo [Online], 30 | 2014, Online since 04 December 2014, connection on 31 July 2021. URL: http://journals.openedition.org/echogeo/14020 ; DOI: https:// doi.org/10.4000/echogeo.14020

This text was automatically generated on 31 July 2021.

EchoGéo est mis à disposition selon les termes de la licence Creative Commons Attribution - Pas d'Utilisation Commerciale - Pas de Modification 4.0 International (CC BY-NC-ND) 


\title{
La pauvreté urbaine à Mexico : une approche de la diversité des colonies populaires
}

\author{
Antonine Ribardière and Jean-François Valette
}

\section{AUTHOR'S NOTE}

Ce travail s'inscrit dans le programme ANR PERISUD « Dynamiques territoriales à la périphérie des métropoles des Suds ", coordonné par J.-L. Chaléard. Dans ce cadre, il a en particulier bénéficié des échanges avec $\mathrm{E}$. Mesclier et $\mathrm{M}$. Piron. Les résultats présentés ici ont fait l'objet d'une présentation au congrès d'août 2012 de l'UGI et ont été intégrés dans la démonstration de la thèse de J.-F. Valette (2014).

1 La désignation de la pauvreté urbaine donne lieu à des débats théoriques riches, en partie spécifiques à chaque contexte national. Il s'agit à la fois de désigner les formes sociales de la pauvreté et les lieux dans lesquels elle se concentre. Dans le contexte français, ces deux dimensions de la pauvreté urbaine sont saisies séparément : à travers la notion d'exclusion ou de précarité d'une part; et au moyen de l'identification de quartiers pris dans des processus de relégation recouvrant largement les «zones urbaines sensibles » et les autres quartiers prioritaires de l'action publique d'autre part. Dans le contexte mexicain, la notion de marginalidad permet au contraire de saisir, dans un même mouvement, les formes sociales et spatiales de la pauvreté urbaine (Fassin, 1996).

2 La marginalidad a été théorisée au cours des années 1970, alors que l'ensemble du continent latino-américain connaissait une urbanisation massive, nourrie par les afflux de populations rurales vers les pôles urbains, dans des contextes économiques nationaux insuffisants pour absorber une telle croissance. Au Mexique, l'analyse des conditions de subsistance de ces populations nouvellement urbaines a fait l'objet de nombreux travaux, notamment ceux des anthropologues O. Lewis (1963) et L. Lomnitz 
(1975). L'interprétation de la marginalidad et des processus qui y conduisent varie selon le modèle théorique de référence : la théorie de la modernisation (Germani, 1980) d'une part et la théorie marxiste de la dépendance (Nun, 1969), d'autre part (Fassin, 1996 ; Delfino, 2012). Dans tous les cas, la marginalidad permet de rendre compte du développement des barrios marginales, ciudades perdidas et colonias populares, quartiers de concentration de la pauvreté nés au moment de l'explosion urbaine, mais aussi et surtout, principale modalité d'expansion physique de la ville de Mexico depuis 1940, notamment dans les zones périphériques où la production de logements impliquait rapidité, mauvaise qualité et illégalité foncière. Les colonias populares ou colonies populaires, sont ainsi l'appellation générique mexicaine donnée aux quartiers populaires, souvent autoconstruits, nés dans l'illégalité foncière et ne disposant pas des services urbains au moment de leur fondation. Dans les années 1970, alors que la ville connaissait une croissance remarquable (la population passe de 3 à 14 millions entre 1950 et 1980), la moitié de la population de Mexico vivait dans ces colonies ${ }^{1}$, qui représentaient elles-mêmes près des deux tiers de la surface urbanisée (Connolly, 2005).

3 La notion de marginalidad est progressivement mobilisée comme une catégorie de l'action publique ${ }^{2}$, mais c'est finalement une autre notion qui s'impose : la marginación. Le CONAPO (Consejo Nacional de Población) propose en effet dans les années 1990 un indice de marginación permettant d'identifier les territoires les plus marginalisés, à l'échelon des localités urbaines et des municipes en 1996 et, en 2005, des unités de recensement - ageb ${ }^{3}$. Dans la présentation qui en est faite par le CONAPO, cet indice s'affranchit du bagage théorique de la marginalidad et vise directement à appuyer la définition et la mise en œuvre des politiques sociales ${ }^{4}$ (Cortés, 2002). L'espace urbain est spécifiquement caractérisé par un indice de marginación urbana. Ces indices sont construits à partir d'un panel d'indicateurs socio-économiques décrivant la population résidente et les logements, renvoyant ainsi aux mesures qui peuvent être faites de la pauvreté multidimensionnelle 5 . Si la dimension spatiale, autrement dit la position des quartiers dans la ville, n'est pas directement intégrée dans l'indice de marginación urbana, les colonies populaires sont toutefois explicitement visées. Il s'agit en effet de cerner la population résidant «en périphérie des villes et dans des zones non urbanisables » (CONAPO, 2005) ${ }^{6}$.

4 Pourtant, l'association entre colonies populaires et pauvreté urbaine ne peut se faire aujourd'hui sans nuances. Les trajectoires de consolidation des vastes ensembles de colonies populaires des années 1970, étudiées notamment à Nezahualcóyotl ou à Valle de Chalco (Hiernaux, Lindón, 2003 ; Vega, 2005), révèlent des processus d'intégration urbaine qui passent par une diversification sociale de la population résidente. L'observation des paysages urbains sur le terrain va dans le même sens. Dans la grisaille et la densité des logements autoconstruits, se nichent des habitations qui donnent à voir des signes d'aisance relative: superficie conséquente, qualité des matériaux de construction, murs peints, équipement automobile, etc. Il n'en reste pas moins que les colonies populaires offrent toujours le visage d'une urbanisation qui s'est développée dans une grande précarité foncière, à l'écart des réseaux de transports de masse, bénéficiant des réseaux électriques au prix de branchements sauvages, et qui reste mal connectée aux réseaux d'adduction et d'évacuation des eaux.

5 Le recensement de population de 2010 est l'occasion d'offrir une image actualisée de la pauvreté urbaine dans la zone métropolitaine de Mexico ${ }^{7}$. Les indicateurs 
sociodémographiques décrivant la population résidente et les logements à l'échelon fin des ageb permettent en effet de proposer une approche systématique des formes et des niveaux de pauvreté à l'échelle de l'ensemble de la ville. Notre démarche, quantitative, se situe dans la lignée des travaux qui ont cherché à mettre en évidence les formes spatiales de la division sociale de l'espace à Mexico, en particulier ceux de R.-M. Rubalcava et M. Schteingart $(1985,2000,2012)$ ou encore ceux d'E. Duhau et A. Giglia (2008). À partir de cette analyse au niveau de l'aire métropolitaine et en nous appuyant sur les types de peuplement tels que définis dans le cadre de l'observatoire de la ville de Mexico - OCIM (Connolly, 2005), nous pourrons apprécier la spécificité des formes de peuplement associées aux colonies populaires et observer les résultats actuels d'un processus de banalisation socio-spatiale lisible depuis une vingtaine d'années dans ces espaces (Ribardière, Valette, 2014). Il ne s'agit donc pas ici de figer les colonies populaires en une catégorie, mais bien au contraire d'interroger leur homogénéité du point de vue de la pauvreté urbaine telle que nous proposons de la saisir. La diversité relative des colonies populaires sera enfin rapportée à la dimension temporelle qui caractérise les trajectoires de consolidation et d'intégration à la ville. L'étude d'un exemple à une échelle fine, reposant cette fois sur une approche qualitative, permettra d'introduire le rôle de deux autres facteurs de différenciation des colonies populaires: les dimensions foncière et politique.

\section{Lire les formes de répartition de la pauvreté dans la ville}

6 Pour caractériser les formes de pauvreté à l'échelle de l'ensemble de la ville, nous adoptons ici une approche de la pauvreté que l'on peut qualifier de substantialiste ${ }^{8}$, en associant un ensemble d'indicateurs décrivant les caractéristiques des populations résidentes. Quatre dimensions classiques de la pauvreté ont été retenues, liées à l'accès à l'éducation, la santé, aux conditions de logement et au niveau de vie ${ }^{9}$. Les indicateurs décrivent des contextes résidentiels à l'échelon de l'ageb: si ce maillage autorise une lecture fine de l'espace urbain, comme tout découpage il impose des limites qui déterminent l'interprétation et la portée des résultats. En particulier, la maille des ageb est construite suivant des critères opérationnels qui l'éloignent de la définition que l'on pourrait attendre d'un quartier urbain. Toutefois, ce maillage reste une référence pour les analyses infra-urbaines au Mexique et autorise ainsi le croisement des sources statistiques avec d'autres sources d'information.

\section{Le choix des indicateurs pour appréhender la pauvreté multidimensionnelle}

7 Un certain nombre des indicateurs retenus recoupent ceux mobilisés par l'indice de marginación urbana du CONAPO : la part des enfants non scolarisés, la part de la population sans couverture sociale, la part des logements dont le sol est en terre battue, la part de ceux ne disposant pas d'une connexion au réseau d'eau ou au réseau d'assainissement.

8 Notre projet étant de décrire des lieux du point de vue des caractéristiques de la population résidente et du niveau de concentration de la pauvreté - et non de cerner la population pauvre elle-même - nous avons ajouté deux indicateurs qui visent à 
témoigner de la présence de classes moyennes et/ou supérieures : la part des diplômés du "supérieur » et la part des logements connectés au réseau Internet. Malgré le développement de l'enseignement supérieur, l'accès aux diplômes reste à Mexico un indicateur pertinent pour appréhender les stratifications sociales, renvoyant non seulement aux structures démographiques, mais également aux inégalités sociales (Didou, Aupetit, 2011). Par ailleurs, si l'usage d'Internet est banalisé, l'existence d'une connexion au sein du logement reste, en 2010 , très discriminante. Une ageb pourra ainsi être appréciée comme constituant un contexte local de pauvreté, si les conditions de vie précaires sont surreprésentées et également, si les signes d'appartenance aux catégories moyennes et supérieures, en termes de niveau d'études et d'équipement, sont sous-représentés ${ }^{10}$.

Enfin, nous avons intégré une dernière dimension de différenciation, relevant davantage du champ de la division sociale de l'espace que de la pauvreté stricto sensu, en tentant de prendre en compte les populations « indigènes ». Le recensement de l'Inegi ne permet de cerner que très imparfaitement les groupes ethniques : est considérée comme "indigène » la population qui parle, au moment de l'enquête, une langue « indigène ». Outre les limites intrinsèques du critère linguistique (Gouëset, 2006), cette définition masque la grande diversité des populations « indigènes » à Mexico, ne seraitce que du point de vue de leur origine géographique (Perraudin, 2011). Dans la ville de Mexico, il n'existe pas de quartier spécifiquement défini sur des bases ethniques et la répartition des populations "indigènes " ne recouvre que partiellement les autres formes d'organisation spatiale de la division sociale (Oehmichen, 2001). Toutefois, le lien qui peut être fait, au moins au niveau national, entre la question de la pauvreté et la question "indigène" justifie que nous prenions en compte cette dimension pour caractériser les formes socio-spatiales de pauvreté ${ }^{11}$ : en $2008,75,7 \%$ de la population « indigène » est touchée par une pauvreté multidimensionnelle ${ }^{12}$.

La comparaison des valeurs prises par ces différents indicateurs dans la zone métropolitaine de Mexico avec celles qui valent dans l'ensemble des aires urbaines d'une part, et à l'échelle du pays d'autre part, offre une mise en perspective succincte de la position de la capitale dans la société mexicaine contemporaine (tableau 1).

Tableau 1 - Les indicateurs retenus. Ordres de grandeur à Mexico et au Mexique

\begin{tabular}{|l|l|l|l|}
\hline Indicateurs & $\begin{array}{l}\text { Part dans } \\
\text { la ZMVM, } \\
2010 \\
\text { (en \%) }\end{array}$ & $\begin{array}{l}\text { Part dans les } \\
\text { localités urbaines* } \\
\text { au Mexique, 2010 } \\
\text { (en \%) }\end{array}$ & $\begin{array}{l}\text { Part au } \\
\text { Mexique, } \\
\text { 2010 (en \%) }\end{array}$ \\
\hline Accès à l'éducation & & & 4,1 \\
\hline $\begin{array}{l}\text { Enfants âgés de } 6 \text { à } 14 \text { ans qui ne vont pas à } \\
\text { l'école }\end{array}$ & 3,2 & 4,4 & 6,9 \\
\hline Personnes analphabètes (15 ans et plus) & 2,5 & 42,5 & 36,3 \\
\hline $\begin{array}{l}\text { Personnes ayant un niveau d'étude moyen } \\
\text { (« post-basique ») ou supérieur'13 (18 ans et plus) }\end{array}$ & 48,0 & & \\
\hline Accès à la santé & & & \\
\hline
\end{tabular}




\begin{tabular}{|c|c|c|c|}
\hline $\begin{array}{l}\text { Personnes ne disposant d'aucune couverture } \\
\text { sociale }\end{array}$ & 38,6 & 32,8 & 33,8 \\
\hline Personnes bénéficiant du Seguro Popular ${ }^{14}$ & 10,0 & 16.9 & 23,3 \\
\hline \multicolumn{4}{|l|}{ Conditions de logement } \\
\hline Nombre moyen de personnes par pièce ${ }^{15}$ & 1,0 & 1,0 & 1,5 \\
\hline Logements dont le sol est en terre battue & 1,8 & 3,6 & 6,1 \\
\hline Logements d'une seule pièce & 6,5 & 6,6 & 7,2 \\
\hline $\begin{array}{l}\text { Logements ne disposant pas d'une connexion au } \\
\text { réseau d'eau (dans le logement ou à proximité) }\end{array}$ & 3,1 & 6,0 & 11,3 \\
\hline Logements ne disposant pas d'assainissement & 0,6 & 2,9 & 9,0 \\
\hline \multicolumn{4}{|l|}{ Niveau d'équipement des logements } \\
\hline $\begin{array}{l}\text { Logements ne disposant pas de radio, } \\
\text { réfrigérateur, lave-linge, automobile, télévision, } \\
\text { ordinateur, téléphone, internet dans le total des } \\
\text { logements particuliers habités - aucun bien }\end{array}$ & 0,4 & 1,0 & 2,8 \\
\hline Logements disposant d'un réfrigérateur & 88,1 & 87,6 & 82,0 \\
\hline Logements disposant d'une connexion internet & 31,9 & 26,6 & 21,3 \\
\hline \multicolumn{4}{|l|}{ Population « indigène " } \\
\hline $\begin{array}{l}\text { Membres d'un ménage dont le chef ou son } \\
\text { conjoint parle une langue " indigène " }\end{array}$ & 3,6 & 5,8 & 9,6 \\
\hline
\end{tabular}

* La « localité urbaine » est une unité de découpage administratif prenant en compte un ensemble urbain bâti continu, à l'intérieur d'une délégation ou municipe, d'une population supérieure à 2500 habitants. En 2010, plus de 86 millions de personnes, soit $77 \%$ de la population totale, résident dans une localité urbaine.

Source : INEGI (2010).

\section{Accès à l'éducation}

11 Mexico occupe une position particulière relativement au reste du pays dans le sens où les indicateurs montrent des niveaux beaucoup moins préoccupants. La nonscolarisation des enfants reste l'exception, largement en deçà de la situation observée dans l'ensemble du pays (urbain ou non). La frange des adultes n'ayant pu accéder à l'éducation reste minoritaire et près de la moitié des 18 ans et plus a atteint un niveau d'étude « post basique » ou supérieur. 


\section{Accès aux soins à travers la protection sociale} plus sélectif à celui témoignant des plus grandes formes de dénuement. En 2010 quasiment un logement sur 3 dispose d'une connexion Internet à Mexico, tandis qu'ils ne sont qu'un sur 5 à l'échelle nationale, et un sur 4 dans l'ensemble des zones urbaines du pays. La présence d'un réfrigérateur renvoie concrètement à un élément important de l'économie alimentaire des ménages et témoigne d'un niveau d'équipement intermédiaire (ce qui n'est plus le cas de la télévision, présente dans plus de $97 \%$ des logements en 2010). Enfin, la part des ménages sans biens est, quant à elle, résiduelle à l'échelle de la ville de Mexico ( $0,4 \%)$. Elle permet toutefois de cerner les ménages les plus démunis et concerne plus de $1 \%$ des logements dans 4 municipes sur 10 de l'État de Mexico.

\section{La place des communautés « indigènes »}

17 Malgré les limites du critère linguistique utilisé dans le recensement, soulignées plus haut, il nous est apparu important de tester ${ }^{17}$, au sein du territoire de la ZMVM, la relation entre espaces de pauvreté et espaces résidentiels des populations " indigènes ». On note que la population qui peut être considérée comme "indigène » du point de vue de la langue est largement minoritaire à Mexico (moins de $4 \%$ des habitants), bien davantage qu'à l'échelon national où elle représente près d'une 
personne sur 10. Ce trait caractéristique des zones urbaines est encore renforcé dans la capitale.

Nous retenons de ces paramètres les signes effectifs d'une grande pauvreté à Mexico. Cette dernière apparaît toutefois résiduelle, étant donnée la faible part des populations concernées (grand dénuement matériel ou précarité du logement). En revanche, certains indicateurs (sécurité sociale, équipement) cernent une pauvreté largement diffusée, qui correspond à une pauvreté de masse, caractéristique des pays émergents.

Ces indicateurs proposent chacun un visage de la cartographie sociale de la ville. Toutefois, ces différentes facettes de l'espace résidentiel ne se recoupent que partiellement: il s'agit donc d'identifier les différentes combinaisons qui structurent l'espace urbain.

\section{La différenciation de l'espace résidentiel par la combinaison des indicateurs de pauvreté}

L'analyse en composantes principales (ACP) constitue une méthode statistique classique pour aborder les covariations spatiales d'un ensemble d'indicateurs. Elle va permettre de révéler les principales associations ou exclusions qui structurent les différenciations entre les 5500 ageb de la zone métropolitaine de Mexico, décrites simultanément par les 14 indicateurs.

21 La composante la plus structurante prend la forme d'une échelle de pauvreté, qui oppose deux types d'ageb. D'un côté, celles caractérisées par la surreprésentation des indicateurs de pauvreté, aussi bien ceux relevant d'une pauvreté extrême, mais résiduelle (analphabétisme et précarité des conditions de logements), que ceux relevant d'une pauvreté plus diffuse (absence de couverture sociale). À l'opposé se positionnent les ageb caractérisées par une surreprésentation des indicateurs témoignant de meilleures conditions de vie (niveau d'équipement des ménages) et d'accès aux études « supérieures ".

Le classement des ageb sur cette échelle de pauvreté résume de manière très synthétique l'ensemble des indicateurs considérés, puisqu'il rend compte de $54 \%$ de l'information ${ }^{18}$ contenue dans les 14 variables.

Une composante secondaire mérite d'être retenue, même si sa puissance synthétique est plus faible $-10 \%$ de l'information. En isolant les surreprésentations des logements en matériau précaire et mal connectés aux réseaux, elle permet de cerner les caractéristiques propres des espaces résidentiels périurbains et ruraux, toute chose égale quant à la position de l'ageb sur l'échelle de pauvreté précédemment définie.

Les composantes suivantes sont plus spécifiques, rendant compte de logiques propres à certains indicateurs et mal restituées dans les deux axes précédents (en particulier, la part des populations considérées comme « indigènes ", des enfants non scolarisés, des ménages sans biens et des logements ne disposant pas de l'assainissement de l'eau).

La prise en compte des quatre premières composantes de la structure de différenciations des unités de recensement induite par les indicateurs de pauvreté permet de retenir les logiques principales, en excluant celles propres à certaines ageb seulement. Une classification ascendante hiérarchique (CAH) appliquée sur ces quatre premières composantes aboutit à une typologie des 5500 ageb en 5 classes, cohérentes et différenciées ${ }^{19}$. 


\section{Formes socio-spatiales de la pauvreté dans les unités de recensement à Mexico}

Les ageb constituent autant de contextes résidentiels, que nous allons caractériser d'une part par leur profil social tel que décrit par les 14 indicateurs de pauvreté et d'autre part, par leur position dans la ville.

L'analyse des profils des différents types obtenus, résumant plus de la moitié des différenciations spatiales entre ageb induites par les variables, témoigne des dimensions de la pauvreté dans la géographie sociale de Mexico (Illustration 1).

Illustration 1. - Profil des 5 types d'ageb

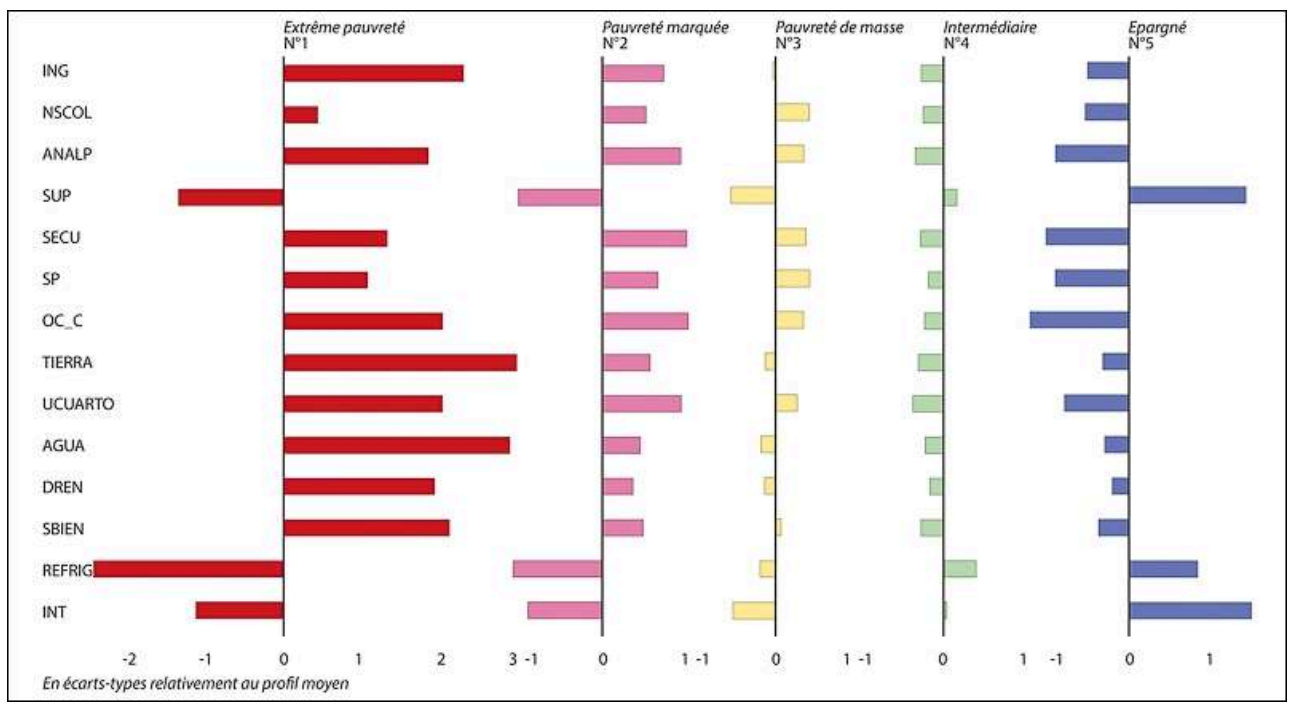

Tableau 2 - Répartition des ageb et de la population résidente dans les 5 classes

\begin{tabular}{|c|c|c|c|}
\hline $\begin{array}{l}\text { Formes socio-spatiales de } \\
\text { pauvreté }\end{array}$ & $\begin{array}{ll}\text { Part } & \text { des } \\
\text { ageb } & \\
\text { (en \%) } & \end{array}$ & $\begin{array}{l}\text { Population en } 2010 \text { (millions } \\
\text { d'habitants) }\end{array}$ & $\begin{array}{l}\text { Part de la population } \\
\text { en } 2010 \\
\text { (en \%) }\end{array}$ \\
\hline $\begin{array}{l}\text { Classe } n^{\circ} 1-\text { "pauvreté } \\
\text { extrême " }\end{array}$ & 3,4 & 0,28 & 1,4 \\
\hline $\begin{array}{l}\text { Classe } n^{\circ} 2-\text { "pauvreté } \\
\text { marquée » }\end{array}$ & 16,5 & 2,85 & 14,6 \\
\hline $\begin{array}{l}\text { Classe } n^{\circ} 3-\text { «pauvreté de } \\
\text { masse » }\end{array}$ & 23,1 & 5,85 & 29,9 \\
\hline $\begin{array}{l}\text { Classe } \quad \mathrm{n}^{\circ} 4 \\
\text { « intermédiaire " }\end{array}$ & 37 & 7,54 & 38,6 \\
\hline Classe $n^{\circ} 5$ - « épargné » & 20 & 3,02 & 15,4 \\
\hline
\end{tabular}



élevés, dans une moindre mesure cependant qu'ils ne le sont dans la classe 1 . En particulier, les indicateurs témoignant d'une précarité des logements propre aux espaces périurbains et ruraux accusent des valeurs moins marquées (logement au sol en terre battue et sans adduction d'eau) ; la part des ménages dépourvus de tout bien est résiduelle. En revanche, d'autres indicateurs de pauvreté sont largement surreprésentés : ils concernent le niveau d'éducation et l'accès à une couverture sociale des populations ; la taille des logements et le niveau d'équipement des ménages. 14,6 \% de la population totale vit dans ces contextes résidentiels qui sont le lieu d'une pauvreté urbaine marquée.

$3130 \%$ de la population de la ZMVM réside dans les ageb du type 3, marquées par une pauvreté de masse : on y observe un niveau de pauvreté plus important qu'en moyenne et qui touche une grande partie de la population. Toutefois, les indicateurs n'atteignent pas des niveaux de surreprésentations observés dans les contextes résidentiels précédents.

8,6 \% de la population réside dans des ageb intermédiaires (type 4) dans lesquelles la pauvreté n'est pas absente, mais moins représentée qu'en moyenne, quel que soit l'indicateur considéré.

Enfin, une frange non négligeable de la population (15,4 \%) vit dans des ageb marquées par la forte surreprésentation de l'accès aux études «supérieures » et des indicateurs d'équipements intermédiaire et supérieur (deux logements sur trois sont connectés à Internet) (type 5). Les indicateurs de pauvreté y sont largement sous-représentés, ce qui amène à considérer ces espaces comme globalement épargnés par les concentrations spatiales de pauvreté. Néanmoins, les signes de pauvreté persistent : en moyenne, le quart de la population résidente ne bénéficie d'aucune protection sociale.

L'inscription spatiale des différents types d'ageb dans la zone métropolitaine de Mexico permet de préciser la nature des contextes résidentiels (illustration 2). 


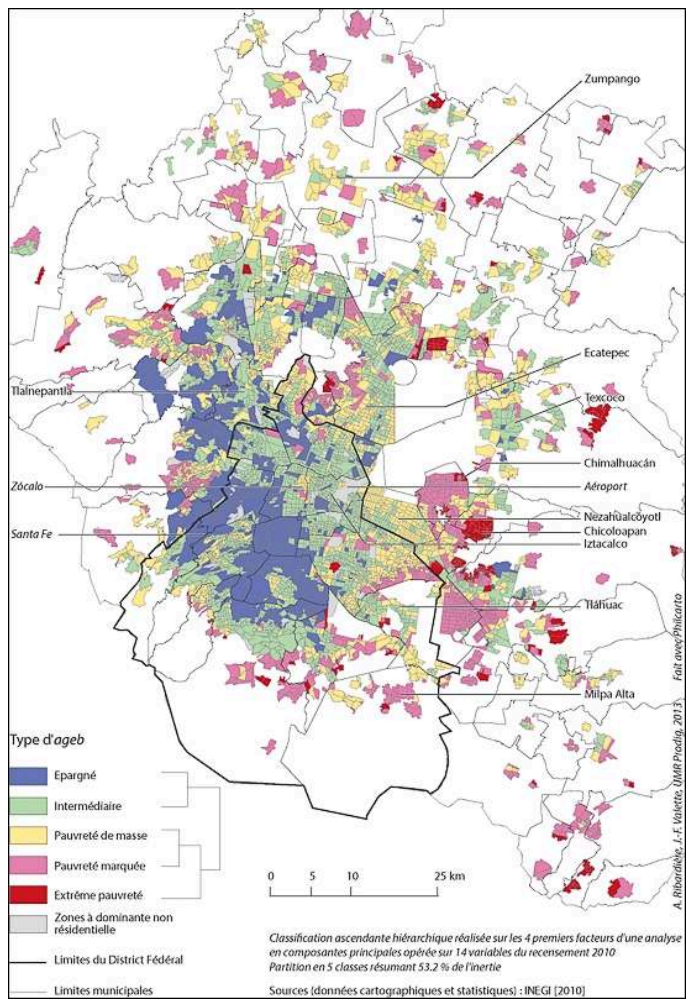

Source : Inegi, 2010. Auteurs : A. Ribardière, J.-F. Valette, 2013

Les ageb marquées par une pauvreté extrême (type 1) sont caractéristiques des fronts d'urbanisation populaire de la grande périphérie - mal reliés au reste de la ville - et des villages en voie de conurbation, comme à Chicoloapan à l'est.

Les ageb du type 2 concernent des espaces plus anciennement urbanisés et davantage consolidés. On les retrouve ainsi dans le prolongement des grandes zones de peuplement populaire et dans les villages périurbains comme celui de Milpa Alta au sud du DF. Ce type d'ageb entre également dans la mosaïque socio-spatiale qui caractérise le centre.

Les ageb témoignant d'une pauvreté de masse sont les plus diffuses, elles s'inscrivent d'une part dans les zones de peuplement populaire en proche périphérie (à Nezahualcóyotl par exemple), bien reliées au reste de la ville et d'autre part, dans des franges périurbaines (comme à Zumpango). Elles constituent par ailleurs une part importante du centre-ville dégradé.

À l'opposé, les ageb les plus épargnées par les concentrations spatiales de pauvreté forment un ensemble compact, dans le péricentre du District Fédéral (DF) et dans ses prolongements sur les collines boisées (Lomas, Santa Fe) de l'ouest de l'agglomération. Quelques ensembles plus isolés se distinguent aussi au nord de l'agglomération.

Les ageb au profil intermédiaire se situent en position de zone tampon, entre les espaces de pauvreté de masse et ceux qui en sont épargnés.

Ces jeux de voisinages et d'éloignement entre profils d'ageb renvoient aux grandes lignes de la géographie sociale de Mexico, marquée par une opposition est-ouest et un gradient centre-périphérie (Rubalcava, Schteingart, 1985, 2000, 2012 ; Duhau, Giglia, 2008 ; Aguilar, Mateos, 2011 ; Tallet, Ribardière, 2011 ; Mesclier et al, 2014). 


\section{Formes socio-spatiales de pauvreté et formes de peuplement : la spécificité des colonies populaires} renvoient, à première vue, aux formes de peuplement selon lesquelles la ville construite: centre-ville, colonies populaires, zones résidentielles pour catégories moyennes et supérieures ou encore grands ensembles de logements sociaux. Des travaux approfondis ont été conduits au Mexique sur ce thème, en particulier par l'OCIM (Connolly, 2005 ; OCIM-SIG). Les enjeux associés à l'identification des formes de peuplement sont particulièrement vifs au regard des questions foncières liées aux colonies populaires.

\section{Un peuplement massif et illégal}

es colonies populaires sont les héritières des asentamientos irregulares ou installations illégales. Il s'agit de l'équivalent mexicain des favelas brésiliennes ou des barrios de rancho vénézuéliens, englobant " ce que le reste de la ville n'est pas " (Connolly, 2003 p.12). Leurs caractéristiques renvoient à des conditions de vie urbaine difficiles sous plusieurs aspects: irrégularité du statut foncier (faute de titre de propriété ou d'autorisation d'urbanisation et/ou violation des règles en vigueur), caractère précaire des constructions, manque de services de base et d'équipements urbains, et inscription dans la durée des dynamiques d'amélioration. L'autoconstruction est un autre élément déterminant de ce type de peuplement (Hiernaux-Nicolas, 1991). Enfin, la colonie populaire revêt une dimension sociologique particulière: la souplesse des relations sociales et les négociations permanentes entre les habitants et les autorités (officielles et officieuses) définissent un ordre social solide, mais aussi fortement lié à des pratiques clientélistes (Duhau, Giglia, 2004, 2008).

Depuis les années 1990, des évolutions profondes sont en cours dans la production urbaine en périphérie où les grands ensembles de logements "d'intérêt social » et l'expansion des villages non intégrés à la ville constituent de plus en plus la forme dominante de croissance de l'agglomération ${ }^{20}$, faisant décliner la part des colonies populaires dans la dynamique d'extension.

Leur poids au sein du peuplement reste toutefois un élément incontournable dans l'analyse des espaces résidentiels (tableau 3). Par la quantité de population qu'elles sont capables de loger et par les enjeux associés aux espaces sur lesquels elles s'étendent, en termes d'irrégularité foncière et de planification urbaine, les colonies populaires renvoient à une composante stratégique de la compréhension du peuplement et des conditions de vie de la population de la zone métropolitaine de Mexico (Connolly, 2003).

41 Pour distinguer les colonies populaires des autres types de peuplement de la ville, nous nous sommes appuyés sur une typologie établie par l'OCIM, que nous avons agrégée et actualisée pour les besoins de cette étude (cf. annexe). Au total, la zone métropolitaine en 2010 est décrite selon cinq grands types de peuplement :

- La ville-centre, construite avant 1929 ;

- Les colonies populaires ;

- Les grands ensembles résidentiels;

EchoGéo, 30 | 2014 
- Les lotissements résidentiels pour classes moyennes et supérieures ;

- Les espaces à dominante non résidentielle.

En 2010, 62 \% des ageb sont classées comme " colonie populaire » selon la définition large que nous avons retenue; 13 millions d'habitants, soit $67 \%$ de la population, y résident (tableau 3).

Tableau 3 - Poids des grands types de peuplement dans la zone métropolitaine de Mexico en 2010

\begin{tabular}{|l|l|l|l|}
\hline Type de peuplement & $\begin{array}{l}\text { Part des ageb en } \\
2010 \text { (en \%) }\end{array}$ & $\begin{array}{l}\text { Part de la population } \\
\text { en 2010 (en \%) }\end{array}$ & $\begin{array}{l}\text { Population (en } \\
\text { millions) }\end{array}$ \\
\hline Ville-centre & 6 & 6 & 1,23 \\
\hline Colonies populaires & 62 & 67 & 13,11 \\
\hline Grands ensembles & 20 & 19 & 3,63 \\
\hline $\begin{array}{l}\text { Résidences catégories } \\
\text { moyennes et supérieures }\end{array}$ & 8 & 6 & 1,26 \\
\hline Non résidentiel & 4 & 2 & 0,31 \\
\hline
\end{tabular}

Sources : OCIM (2005), ajustements propres pour 2010, INEGI (2010).

\section{Croisement des deux typologies}

Le croisement entre ces deux typologies met en évidence les associations entre les ageb identifiées comme les plus épargnées et les types de peuplement relevant des grands ensembles et des quartiers résidentiels moyens et supérieurs (tableau 4). On observe également l'association quasi exclusive entre les contextes résidentiels les plus marqués par la pauvreté et les types de peuplement relevant de la catégorie des colonies populaires. Enfin, les ageb intermédiaires du point de vue de la pauvreté correspondent à des types de peuplement plus variés - pour un tiers, aux grands ensembles et pour plus de la moitié aux colonies populaires - et constituent un signe fort de la diversité des colonies populaires.

Tableau 4 - Décomposition des classes socio-spatiales de pauvreté selon les différents types de peuplement (profils en colonne)

\begin{tabular}{|l|l|l|l|l|l|l|}
\hline $\begin{array}{l}\text { Type de } \\
\text { peuplement/ } \\
\text { Classe }\end{array}$ & $\begin{array}{l}\text { Classe } \mathrm{n}^{\circ} 1 \\
\text { «pauvreté } \\
\text { extrême » }\end{array}$ & $\begin{array}{l}\text { Classe } \mathrm{n}^{\circ} 2 \\
\text { «pauvreté } \\
\text { marquée » }\end{array}$ & $\begin{array}{l}\text { Classe } \mathrm{n}^{\circ} 3 \\
\text { «pauvreté } \\
\text { de masse » }\end{array}$ & $\begin{array}{l}\text { Classerintermédiaire » } \\
\text { « }\end{array}$ & $\begin{array}{l}\text { Classe } \mathrm{n}^{\circ} 5 \\
\text { "épargné » }\end{array}$ & Total \\
\hline Ville-centre & 0 & 0,7 & 1,4 & 5,6 & 16,4 & 6 \\
\hline $\begin{array}{l}\text { Colonies } \\
\text { populaires }\end{array}$ & $\mathbf{9 5 , 2}$ & $\mathbf{9 2 , 5}$ & $\mathbf{9 0 , 2}$ & $\mathbf{5 4 , 9}$ & $\mathbf{1 3 , 6}$ & $\mathbf{6 2}$ \\
\hline Grands ensembles & 0 & 1,4 & 6,2 & 33,4 & 32 & 20 \\
\hline
\end{tabular}




\begin{tabular}{|l|l|l|l|l|l|l|}
\hline $\begin{array}{l}\text { Résidences } \\
\text { catégories } \\
\text { moyennes et } \\
\text { supérieures }\end{array}$ & 0 & 0,1 & 0,2 & 2,6 & 33,2 & 8 \\
\hline Non résidentiel & 4,8 & 5,3 & 2,1 & 3,6 & 4,9 & 4 \\
\hline Total & 100 & 100 & 100 & 100 & 100 & 100 \\
\hline
\end{tabular}

Pourcentage en colonne : $95.2 \%$ des ageb de la classe $n^{\circ} 1$ relèvent du type « colonie populaire ».

\section{Tableau 5 - Décomposition des types de peuplement selon les différentes classes socio-spatiales} de pauvreté (profils en ligne)

\begin{tabular}{|l|l|l|l|l|l|l|}
\hline $\begin{array}{l}\text { Type de } \\
\text { peuplement/ } \\
\text { Classe }\end{array}$ & $\begin{array}{l}\text { Classe } \mathrm{n}^{\circ} 1 \\
\text { «pauvreté } \\
\text { extrême » }\end{array}$ & $\begin{array}{l}\text { Classe } \mathrm{n}^{\circ} 2 \\
\text { «pauvreté } \\
\text { marquée » }\end{array}$ & $\begin{array}{l}\text { Classe } \mathrm{n}^{\circ} 3 \\
\text { «pauvreté } \\
\text { de masse » }\end{array}$ & $\begin{array}{l}\text { Classe } \mathrm{n}^{\circ} 4 \\
\text { «intermédiaire» }\end{array}$ & $\begin{array}{l}\text { Classe } \\
\mathrm{n}^{\circ} 5 \\
\text { «épargné» }\end{array}$ & Total \\
\hline Ville-centre & 0,0 & 1,9 & 5,7 & 35,7 & 56,8 & 100 \\
\hline Colonies populaires & 5,2 & 24,5 & 33,4 & 32,6 & 4,3 & 100 \\
\hline Grands ensembles & 0,0 & 1,2 & 7,0 & 60,5 & 31,3 & 100 \\
\hline $\begin{array}{l}\text { Résidences } \\
\text { catégories } \\
\text { moyennes } \\
\text { supérieures }\end{array}$ & 0,0 & 0,2 & 0,5 & 12,4 & 86,9 & 100 \\
\hline Non résidentiel & 4,3 & 22,9 & 12,4 & 34,8 & 25,7 & 100 \\
\hline Total & 3,4 & 16,5 & 23,1 & 37,0 & 20,0 & 100 \\
\hline
\end{tabular}

Pourcentage en ligne : 5,2 \% des ageb du type « colonie populaire » relèvent de la classe $n^{\circ} 1$

La composition des colonies populaires n'est pas tranchée : au contraire, ce groupe d'ageb se partage à parts égales entre les contextes résidentiels les plus marqués par la pauvreté (classes 1 et 2), ceux relevant d'une pauvreté de masse (classe 3) et enfin, les intermédiaires (classe 4). Cette diversité laisse une place, même minoritaire, aux ageb les plus épargnées (classe 5).

L'hétérogénéité de la ville-centre, considérée ici dans son acceptation large, apparaît moindre, puisqu'elle se cantonne quasi exclusivement à la combinaison d'ageb intermédiaires et épargnées.

Les ageb catégorisées comme des grands ensembles apparaissent relativement homogènes, composées dans leur majorité par des quartiers intermédiaires et pour un tiers d'entre eux, d'ageb épargnées. 
En revanche, l'homogénéité de l'espace résidentiel des catégories moyennes et aisées apparaît spectaculaire : cette catégorie n'admet quasiment aucune ageb marquée par la pauvreté. À Mexico comme ailleurs, les logiques d'entre soi qui sont à l'origine du peuplement de ces quartiers résidentiels sont les vecteurs d'une homogénéité sociale plus grande que les logiques plus largement subies qui déterminent les trajectoires résidentielles des catégories populaires.

\section{La diversité des colonies populaires}

Une approche des formes de pauvreté à l'échelle des 5500 ageb qui composent la zone métropolitaine de Mexico permet ainsi de mettre en évidence la grande diversité qui caractérise les colonies populaires. Afin de mieux cerner cette hétérogénéité, nous focalisons maintenant sur les quelques 3430 ageb identifiées comme colonies populaires.

La carte ci-dessous (illustration 3) présente la typologie des formes de pauvreté appliquée uniquement sur les ageb relevant de la catégorie des colonies populaires ${ }^{21}$; elle permet d'appréhender les formes spatiales induites par la diversité qui caractérise ce type de peuplement.

51 Les cinq formes de pauvreté s'organisent dans l'espace de façon remarquable, depuis les ageb les plus centrales qui sont également les plus épargnées par la pauvreté, jusqu'aux ageb les plus périphériques, marquées par une pauvreté extrême. Ce gradient s'observe à différentes échelles.

Illustration 3 - Formes socio-spatiales de pauvreté au sein des colonies populaires

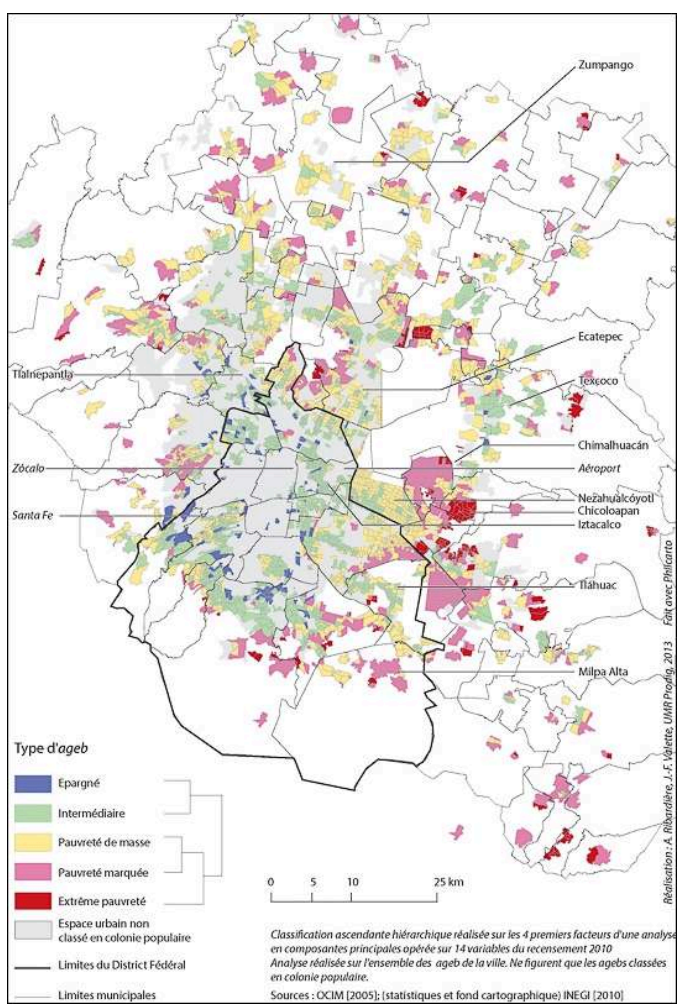

Sources : OCIM, 2005 ; statistiques et fonds cartographiques : INEGI, 2010. Auteurs : A. Ribardière, J.F. Valette, 2013. 
52 À l'échelle de l'agglomération, un gradient systématique apparaît dans la couronne périphérique, au nord, à l'est et au sud de la ville. De portée faible dans le sud du DF, en raison des contraintes physiques et réglementaires qui entravent l'étalement urbain (sols de conservation), il s'étale sur plus de $20 \mathrm{~km}$ à l'est, débordant largement sur l'État de Mexico. Un transect depuis l'aéroport jusqu'aux collines orientales illustre la succession ordonnée des contextes socio-spatiaux de pauvreté. Les ageb péricentrales au niveau de l'aéroport (Iztacalco) renvoient à des contextes résidentiels intermédiaires, qui sont également ceux de la frange la plus ancienne de Nezahualcóyotl, dans l'État de Mexico. L'essentiel du municipe toutefois relève de contextes marqués par une pauvreté de masse. Plus à l'est, les ageb plus récemment urbanisées du municipe de Chimalhuacán sont davantage marquées par la pauvreté. Enfin, aux limites de la ville, à Chicoloapan, s'étendent les ageb d'un niveau de pauvreté extrême. Un tel agencement des contextes socio-spatiaux de pauvreté se retrouve au nord, dans le municipe d'Ecatepec ou encore dans celui de Tlalnepantla.

L'organisation en gradient se lit également à l'échelle des centres secondaires, en périphérie de l'agglomération. Texcoco en est un bon exemple: le centre historique apparaît comme épargné par la concentration de pauvreté, qui augmente à mesure que l'on s'en éloigne.

Enfin, des gradients apparaissent à une échelle plus locale encore, comme à Tláhuac, rappelant la force de cette logique spatiale spécifique aux colonies populaires, qui exclut les contacts brutaux entre les extrêmes et ce, malgré l'hétérogénéité qui pourrait être observée à un échelon encore plus fin, celui de l'îlot, voire du logement.

La structuration spatiale en gradient renvoie d'une part au processus de peuplement propre des colonies populaires - de proche en proche - et d'autre part, à sa dimension temporelle. Le processus de peuplement s'accompagne ici d'un rattrapage progressif des zones les plus anciennement urbanisées relativement au reste de la ville (Schteingart, 1997). Cette banalisation s'exprime aussi bien dans le profil social des populations résidentes que dans la consolidation des logements et l'accès aux réseaux urbains (Ribardière, Valette, 2014).

Le modèle de la consolidation, proposé dès les années 1960 par Turner ${ }^{22}$, est particulièrement lisible sur le transect oriental décrit plus haut. Les colonies urbanisées autour de l'aéroport dans les années 1950 échappent aujourd'hui aux concentrations de pauvreté. La partie du municipe de Nezahualcóyotl urbanisée dans les années 1960 et 1970 se partage entre des contextes résidentiels intermédiaires et d'autres marqués par une pauvreté de masse; les prolongements des années 1980 sont aujourd'hui caractérisés par une pauvreté marquée et les colonies les plus récentes, urbanisées depuis les années 1990, restent les espaces d'une pauvreté extrême.

La focalisation sur une échelle très locale d'observation va permettre de valider ce modèle, mais également de proposer d'autres paramètres d'évolution.

\section{Nuancer les trajectoires en gradient : l'exemple de Santiago Zapotitlán, Tláhuac}

L'exemple d'un quartier du sud-est du District Fédéral met en lumière à la fois le rôle de l'ancienneté du peuplement et des processus de consolidation, divers en qualités et en vitesses. Cet exemple, appréhendé de manière empirique lors de séjours de terrain 
entre 2009 et 2011, permet également de souligner le poids de la dimension politique et des jeux d'acteurs dans les trajectoires d'évolution des colonies populaires (Valette, 2014a).

Ce quartier est une extension d'un noyau villageois aujourd'hui complètement intégré au reste de la ville. Les ageb qui le composent sont classées sans exception en colonie populaire. Toutefois, on y observe de manière très nette le gradient des formes sociospatiales de la pauvreté souligné plus haut, ici orienté nord-sud (illustration 4). À proximité de l'avenue Tláhuac, les ageb les plus anciennement urbanisées sont classées comme intermédiaires (classe 4). Celles plus au sud sont caractérisées progressivement par une pauvreté de masse, puis par une pauvreté marquée et enfin, dans les zones toujours illégales en 2011 et urbanisées depuis une vingtaine d'années, par une pauvreté extrême.

\section{Illustration 4 - Gradient de pauvreté et rythmes de consolidation}

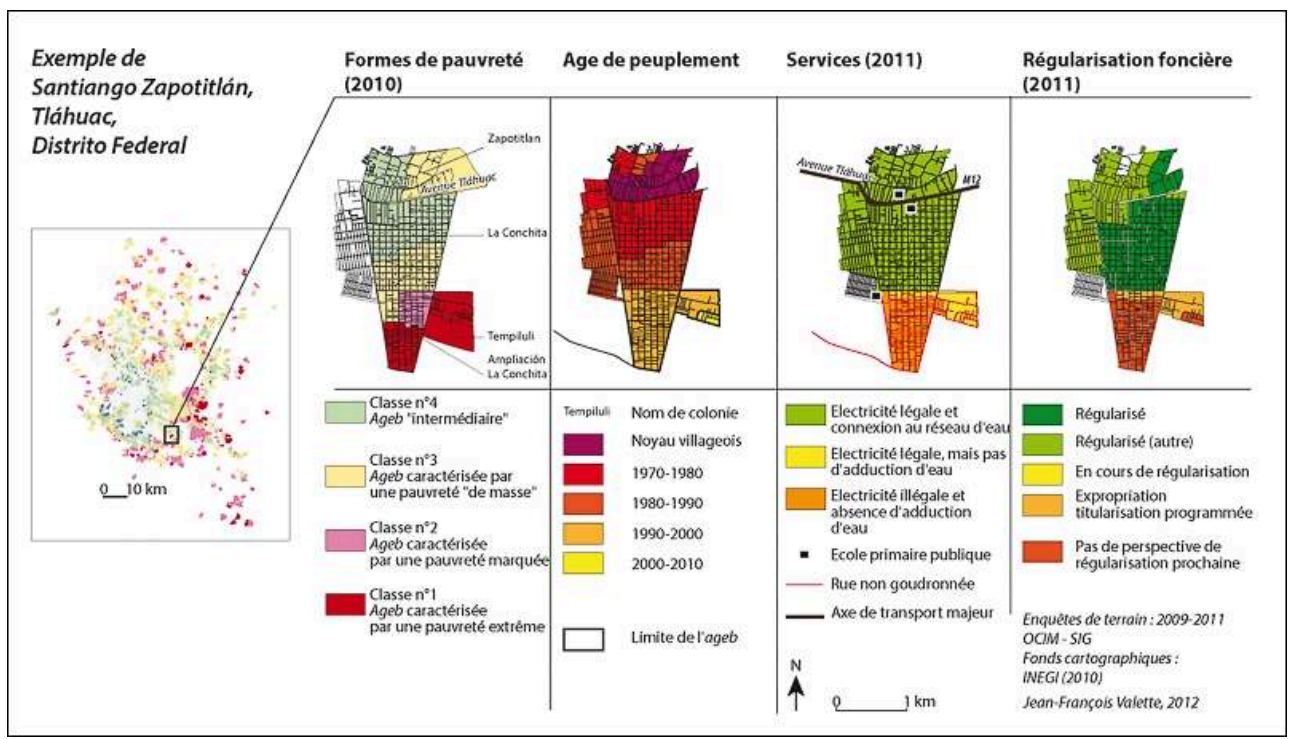

Sources : enquêtes de terrain, 2009-211 ; OCIM-SIG ; Fonds cartographiques : INEGI, 2010

a colonie La Conchita, au nord, est peuplée depuis les années 1970 et a bénéficié d'une régularisation des services urbains (eau et électricité correctes et légales) en 1992. À Ampliación La Conchita, au sud, les services manquent et seule une petite partie de la colonie semble en voie de régularisation. Si l'âge du peuplement paraît expliquer en partie ce gradient, cet élément se révèle insuffisant.

Le statut foncier constitue une autre explication de ces écarts ${ }^{23}$. La première colonie a bénéficié d'un changement de statut foncier favorisant la régularisation, la propriété du sol y est aujourd'hui complètement privée. La deuxième colonie est toujours sous le régime collectif de l'ejido, qui caractérise des terres a priori inconstructibles. La régularisation foncière reste inenvisageable à court terme; du point de vue des habitants, la propriété des terrains et des logements n'est pas juridiquement reconnue.

Enfin, la dimension politique est elle aussi essentielle pour comprendre les trajectoires. Le rôle de l'action collective est en effet majeur dans les processus de consolidation et de régularisation foncière. Ainsi, à Ampliación La Conchita, l'organisation de l'action collective est marquée par des divisions internes la rendant peu efficace, tandis qu'à Tempiluli, plus récemment urbanisé, la situation semble en meilleure voie, notamment 
parce qu'une association est particulièrement active. La pression exercée sur les services municipaux a ainsi permis l'électrification de la colonie; le dialogue instauré avec les autorités locales a abouti à la programmation de la régularisation foncière. Les processus de régularisation renvoient alors à l'analyse de mécanismes clientélistes anciens, particulièrement bien ancrés, tels que mis en évidence par Gilbert et Ward (1985).

63 L'image d'une ville informelle, fondée sur des négociations permanentes, constitue ainsi une entrée pour comprendre les modes d'urbanisation populaires, mais aussi pour appréhender l'insertion politique des groupes d'acteurs issus d'espaces « marginaux » à l'origine. On retrouve alors la définition de colonie populaire proposée par Duhau et Giglia, qui l'entendent comme "la ville négociée » (Duhau, Giglia, 2004). Les négociations, au carrefour des intérêts individuels (ou relevant de la sphère privée) et collectifs (ou relevant de la sphère publique), portent alors sur les actions à mener par les habitants au sein de leur contexte résidentiel: participation politique, travaux d'équipement collectif dans l'espace public, ou encore tolérance du statu quo sont à l'origine d'un contrôle social d'une part, des évolutions différenciées de la consolidation des colonies populaires d'autre part. La variabilité de ces règles tacites participe ainsi de la complexité des formes et des processus de consolidation et par-là, de l'hétérogénéité des formes socio-spatiales de la pauvreté dans ces espaces.

\section{Conclusion}

Mexico reste marquée par une pauvreté de masse, qui se concentre dans les formes de peuplement nées dans l'illégalité foncière et urbanistique: les colonies populaires. Larges portions de l'agglomération qui se sont urbanisées en marge de la ville légale, par les résidents eux-mêmes sans qu'ils disposent de titres de propriété reconnus, elles constituent la forme majeure de l'expansion urbaine depuis un demi-siècle.

Réunissant en 2010 les deux tiers des habitants de la zone métropolitaine, cette forme de peuplement est loin d'être homogène. Décrites sous l'angle de la pauvreté multidimensionnelle, les colonies populaires apparaissent comme des espaces de pauvreté, sans être les seuls concernés et sans qu'elles puissent s'y réduire. Plus encore, à travers la grille retenue dans cette étude des grandes formes de peuplement, elles constituent la forme la plus hétérogène. La diversité s'organise de manière spécifique : sous la forme de gradients qui renvoient aux processus de peuplement et de rattrapage successif, propres aux colonies populaires. Une analyse à un échelon plus fin que celui des ageb permettrait de mettre en évidence des formes plus locales de microségrégations (Dureau, 2000). Les explorations conduites sur la ville de Mexico tendent à mettre en évidence des ruptures locales fortes, sans toutefois remettre en question la structuration en gradients (Valette, 2014b).

Ces formes spatiales récurrentes, à différentes échelles, valident le modèle de la consolidation. Les colonias populares doivent être analysées comme un type d'habitat socialement et spatialement évolutif, en général de manière " ascendante ", hétérogène et non linéaire. Ce profil social mobile du quartier populaire (par rapport à la stratification socio-spatiale et à l'intégration à la ville), pourtant au cœur de la question de l'évolution de ces espaces, est masqué par l'appellation générique «colonie populaire ». 
67

lanalyse d'un exemple à une échelle politique pour caractériser la diversité des colonies populaires. Une telle dimension est difficilement appréhendable de manière systématique dans les analyses de la pauvreté urbaine, en raison de la nature des données - par essence qualitatives - nécessaires à son exploitation. Elle reste néanmoins indispensable pour saisir les processus de régularisation intimement liés à la diversification du profil social des populations résidentes.

\section{BIBLIOGRAPHY}

Aguilar A.G., Mateos P., 2011. Diferenciación sociodemográfica del espacio urbano de la Ciudad de México. EURE, n¹10, vol. 37, p.5-130 [en ligne] : http://www.eure.cl/wp-content/uploads/ 2011/04/EURE_110_01_AGUILAR_MATEOS.pdf

Bey M., 2008. Le programme social Progresa-Oportunidades au Mexique. De vieilles recettes pour un nouveau modèle. Revue Tiers Monde, n 196, p. 881-900.

Blanchon D., 2010. L'eau, une ressource menacée ? La Documentation photographique, $\mathrm{n}^{\circ}$ 8078, 64 p.

Boltvinik J. Hernández Laos E., 1999. Conceptos y Medidas de Pobreza. Pobreza y Distribución del Ingreso en México, Siglo XXI Editores México, p. 30-80.

Capron G., 2003. Proximité spatiale, distance sociale dans les Amériques. Les résidences sécurisées, une modalité de la nouvelle ségrégation urbaine? Rapport final du programme PUCA, $172 \mathrm{p}$.

CONAPO, 2005. Índice de marginación urbana. México, 237 p.

CONEVAL, 2009. Metodología para la medición multidimensional de la pobreza en México. Consejo Nacional de Evaluación de la Política de Desarrollo Social, México, 119 p.

Connolly P., 2003. Urban Slums Reports: The Case of Mexico City, Mexico. UAM-A, Mexico City, 30 p.

Connolly P., 2005. Tipos de poblamiento en la Ciudad de México, reporte de investigación. Serie Metodología, OCIM-SIG, UAM-A, México, 30p.

Cortés F., 2002. Consideraciones sobre marginalidad, marginación, pobreza y desigualdad en la distribución del ingreso. Papeles de población, n³1, UAEM, Toluca, p. 9-24.

Damián A., 2008. La construcción del dato de pobreza. In Figueroa Campos B. (dir.), El Dato en Cuestión, análisis de las cifras sociodemográficas, México D.F., Colegio de México, p. 691-740.

Delfino A., 2012. La noción de marginalidad en la teoría social latinoamericana: surgimiento y actualidad. Universitas Humanística, $\mathrm{n}^{\circ}$ 74, p. 17-34.

Destremau B., Salama P., 2002. Mesures et démesure de la pauvreté. Paris, PUF, 163 p.

Didou Aupetit S., 2011. Inégalités scolaires et ethnicisation dans l'enseignement supérieur au Mexique. Autrepart, n5 59, p. 19-35.

Duhau E., Giglia Á., 2004. Conflictos por el espacio y orden urbano. Estudios Demográficos y Urbanos, vol. 19, n² 2, Colegio de México, México, p. 257-288. 
Duhau E., Giglia A., 2008. Las reglas del desorden: habitar la metrópoli. UAM-A, México, 570 p.

Dureau F., 2000. Les nouvelles échelles de la ségrégation à Bogota. In Dureau F., Dupont V., Lelièvre E., Lévy J-P., Lulle T., Métropoles en mouvement. Une comparaison internationale, Anthropos, IRD, Collection Villes, Paris, p. 247-256.

Fassin D., 1996. Exclusion, underclass, marginalidad : Figures contemporaines de la pauvreté urbaine en France, aux États-Unis et en Amérique latine. Revue Française de Sociologie, vol. 37, $\mathrm{n}^{\circ}$ 1, p. 37-75.

François J.-C., Ribardière A., Fleury A., Mathian H., Pavard A., Saint-Julien T., 2011. Les disparités de revenus des ménages franciliens. Analyse de l'évolution 1999-2007. Rapport final, DREIA Ile-de-France, UMR Géographie-cités, 93 p.

Germani G., 1980. El concepto de marginalidad. Buenos Aires, Nueva visión.

Gouëset V., 2006. Comment définir les catégories ethniques ?In Dureau F., Gouëset V., Mesclier E., Géographies de l'Amérique latine, Rennes, PUR, p. 91-110.

Gutiérrez-Pulido H., Gama-Hernández V., 2010. Limitantes de los índices de marginación de Conapo y propuesta para evaluar la marginación municipal en México. Papeles de Población, vol. $16, \mathrm{n}^{\circ} 66, \mathrm{p} .227-257$.

Hiernaux D., 1991. La Autoconstrucción de la Vivienda en el Área Metropolitana de la Ciudad de México. Revista Interpretaciones, p. 58-73.

Hiernaux D., Lindón A., 2003. Pratiques et stratégies résidentielles dans la Vallée de Chalco, périphérie de Mexico. Autrepart, n²5, p. 123-136.

Hiernaux D., 2005. La inserción urbana de la población indígena en Ciudad de México. In Bodson P., Cordero A., Carrasco P., Goulet J. (eds.), Vivir en la ciudad. Lo cotidiano de la inserción urbana en México, América Central y el Caribe, Flacso, San José Costa Rica, p. 253-272.

Lewis O., 1961. Les enfants de Sanchez. Autobiographie d'une famille mexicaine. Gallimard, 644 p. Lomnitz L., 1975. Como sobreviven los marginados. Siglo XXI, 229 p.

Mesclier E., Piron M., Ribardière A., Valette J-F., 2014. Divisions de l'espace résidentiel : une comparaison entre deux métropoles du Sud, Lima et Mexico. In Chaléard J.-L. (dir.), Métropoles au Sud. Le défi des périphéries?, Karthala, Paris, p. 81-100.

Nun J., 1969. Sobrepoblación relativa, ejercito industrial de reserva y masa marginal. Revista latinoamericana de Sociología, $\mathrm{n}^{\circ}$ 2, p. 178-236.

OCIM-SIG (Observatorio de la Ciudad de México- Sistema de Información Geográfica) [en ligne]: http://www.ocim.azc.uam.mx/.

Oehmichen C., 2001. Espacio urbano y segregación étnica en la ciudad de México. Papeles de población, $\mathrm{n}^{\circ}$ 28, p. 181-197.

Paugam S., 2005. Les formes élémentaires de la pauvreté. Paris, PUF, 276 p.

Perraudin A., 2011. Ethnicité et mobilité. L'expérience migratoire des Indiens mexicains, entre migration interne et internationale. Thèse de sociologie, EHESS, $604 \mathrm{p}$.

PNUD. , 2010. Rapport complet sur le développement humain 2010. New-York, Publications des Nations Unies, $254 \mathrm{p}$. 
Ribardière A., Bonerandi-Richard E., Martin M., Merchez L., 2014. La pauvreté dans l'espace européen : grilles de lecture. In Bonerandi-Richard E. et Boulineau E. (coord.), La pauvreté en Europe. Une approche géographique, PUR, coll. Géographie sociale, p. 17-32.

Ribardière A., Valette J-F., 2012. The colonias populares of Mexico City: sites of marginality? $32^{\text {ème }}$ Colloque International de Géographie, International Geographical Union, Commission « Marginalisation, Globalisation and Regional and Local Responses », Cologne, 29 août 2012.

Ribardière A., Valette J.-F., 2014. Évolution de la division sociale de l'espace urbain à Mexico. Approche à partir des structures démographiques et de l'accès au diplôme (1990-2010). Problèmes d'Amérique latine, n 90, p. 99-126.

Rubalcava R.M., Schteingart M., 1985. Diferenciación socio-espacial intraurbana en el área metropolitana de la ciudad de México. Estudios Sociológicos, n 3, p. 481-514.

Rubalcava R.M., Schteingart M., 2000. Segregación socio-espacial en el área metropolitana de la Ciudad de México. In Garza G. (coord.), La Ciudad de México en el fin del segundo milenio, México, Colegio de México, Gobierno del Distrito Federal, p. 287-294.

Rubalcava R.M., Schteingart M., 2012. Ciudades divididas, Desigualdad y segregación social en México. El Colegio de México, México, 214 p.

Schteingart M., 1997. Urbanización popular y regularización de la tenencia de la tierra. Dos casos en municipios conurbanos del Estado de México. In Azuela A., Tomas F. (dir.), Acceso de los pobres al suelo urbano, UNAM, México, p. 277- 296.

SEDESOL (Secretaria de desarrollo social), CONAPO (Consejo Nacional de población), INEGI (Instituto Nacional de Estadística, Geografía e Informática), 2012. Delimitación de las zonas metropolitanas de México 2010, México.

Sen A., 1987. On Ethics and Economics. Oxford, Blackwell, 148 p.

Tallet B., Ribardiere A., 2011. Mexico, Atlas/Mégapoles, ed. Autrement, Paris, 88 p.

Valette J.F., 2014a. La régularisation territoriale des quartiers populaires de Mexico. Une approche des rapports de pouvoir dans la maturation des périphéries. In Chaléard J.-L. (dir.), Métropoles au Sud. Le défi des périphéries?, Karthala, Paris, p. 369-384.

Valette J.-F., 2014b. Mobilités et ancrages dans les quartiers populaires de la périphérie de Mexico : une approche de la maturation urbaine. Thèse de doctorat de géographie, Université Paris 1, 955 p.

Vega A. L., 2005. Mutations du système de logement dans un quartier à développement spontané après régularisation de la propriété de la terre. Cas de Netzahualcóyotl, dans la banlieue est de Mexico. Thèse de doctorat en urbanisme, Université Paris 12, IUP, 352 p.

\section{APPENDIXES}

\section{Les types de peuplement dans l'agglomération de Mexico en 2010}

La caractérisation des types de peuplement à l'échelon de l'ageb en 2010 a été élaborée à partir du croisement de plusieurs sources :

- la typologie de peuplement de l'agglomération en 2000 réalisée par l'OCIM à l'échelon de l'ageb (Connolly, 2005, p.15-19),

- une actualisation propre pour 2010. 
Pour les besoins de cette étude, nous avons simplifié la lecture de l'espace urbain de l'OCIM en 2000 afin, d'une part, de cerner plus particulièrement l'ensemble " colonie populaire » et afin, d'autre part, d'assurer l'actualisation de l'information. On retient ici une acception large des colonias populares, relevant des caractéristiques communes qui les définissent : irrégularité initiale et caractère progressif de la construction des logements.

\section{Agrégation des types de peuplement de l'OCIM de 2000}

En 2000, l'OCIM établit 12 types de peuplement différents. Pour 2010, nous avons agrégé ces 12 types en 5 types principaux d'ageb (cf. tableau A et carte A).

\section{Tableau A. Agrégation de la typologie de l'OCIM}

\begin{tabular}{|c|c|c|c|}
\hline & Type en 2000 (OCIM) & & Type agrégé en 2010 \\
\hline 1 & «Ville coloniale » $($ de 1793$)$ & \multirow{2}{*}{ A } & \multirow{2}{*}{ Ville-Centre } \\
\hline 2 & «Ville centrale » construite entre 1820 et 1929 & & \\
\hline 3 & $\begin{array}{l}\text { Centres villageois principaux (existants avant 1929) } \\
\text { aujourd'hui intégrés au tissu urbain }\end{array}$ & \multirow{4}{*}{ B } & \multirow{4}{*}{ Colonie populaire } \\
\hline 4 & $\begin{array}{l}\text { Villages périphériques qui se sont développés après } \\
1929 \text { et aujourd'hui intégrés au tissu urbain }\end{array}$ & & \\
\hline 5 & Colonias populares & & \\
\hline 6 & Villages périphériques non intégrés au tissu urbain & & \\
\hline 6 & $\begin{array}{l}\text { «Ensembles de logements » souvent en série et dont la } \\
\text { construction est liée au secteur public }\end{array}$ & C & Grand ensemble \\
\hline 7 & Lotissements résidentiels pour classes moyennes & \multirow{2}{*}{$\mathrm{D}$} & \multirow{2}{*}{$\begin{array}{l}\text { Espace résidentiel pour catégories } \\
\text { moyennes-supérieures }\end{array}$} \\
\hline 8 & Lotissements résidentiels pour classes supérieures & & \\
\hline 10 & Espaces dont l'usage principal n'est pas résidentiel & \multirow{3}{*}{ E } & \multirow{3}{*}{$\begin{array}{l}\text { Espace à dominante non } \\
\text { résidentielle }\end{array}$} \\
\hline 11 & Espaces non résidentiels & & \\
\hline 12 & Zones non urbanisées & & \\
\hline
\end{tabular}

Carte A. Grands types de peuplement dans la zone métropolitaine de Mexico en 2010 


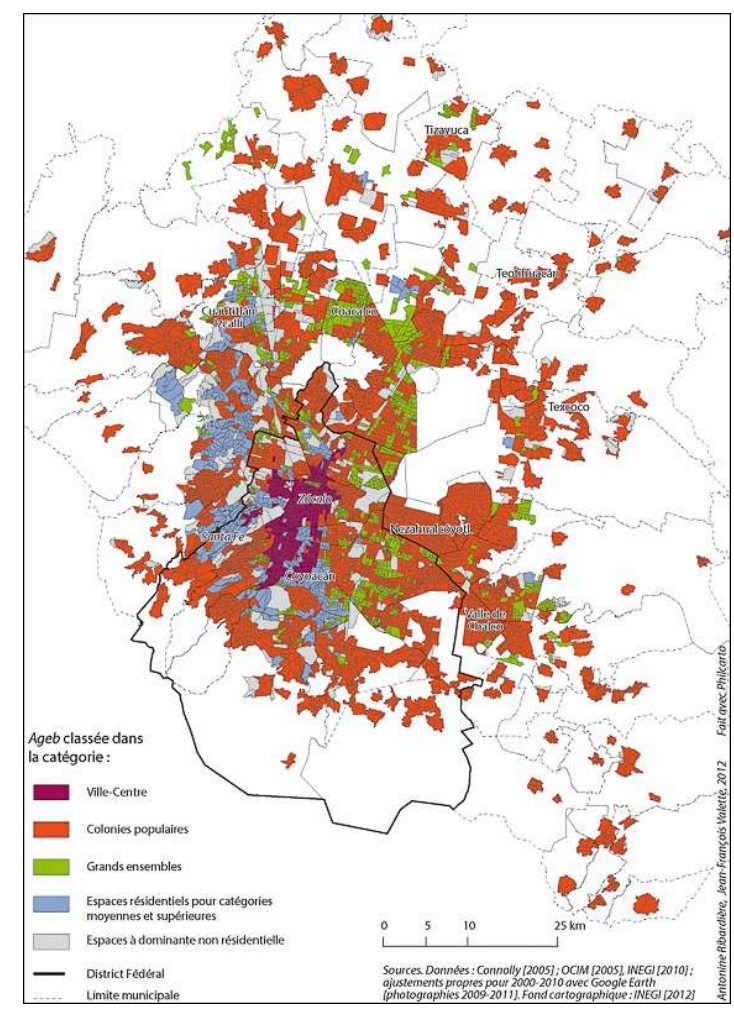

Affectation du type de peuplement en 2010 et modifications ponctuelles

Les espaces urbains et le maillage de l'ageb étant différents entre 2000 et 2010, il a été nécessaire d'affecter un type de peuplement aux unités géostatistiques qui n'en possédaient pas en 2000 - les nouvelles ageb identifiées entre 2000 et 2010 étant liées, d'une part à l'urbanisation de zones non prises en compte en 2000, d'autre part à des modifications occasionnelles entrainant un redécoupage des mailles. En outre, une vérification systématique des zones identifiées comme " non résidentielles » en périphérie en 2000 a été effectuée, celles-ci étant les plus susceptibles d'avoir changé (en termes d'urbanisation) pendant la décennie écoulée.

L'actualisation de la typologie de l'OCIM s'est opérée sur 923 ageb, à partir d'analyses de photographies aériennes ou satellitaires via Google Earth et d'enquêtes de terrain répétées entre 2009 et 2011.

\section{NOTES}

1. La colonie résidentielle est un terme d'urbanisme apparu à la fin du 19e siècle et désignant ce que nous appelons communément le «quartier». La colonie s'affiche comme un projet urbain opposé au barrio dans le sens où, contrairement à ce dernier, connoté d'une construction « spontanée » ou « traditionnelle », elle serait la continuation structurée du reste du tissu urbain (par respect de la trame orthogonale notamment). La colonie sera plus tard utilisée pour faire référence au lotissement d'un quartier, même irrégulier.

2. En particulier, on pense ici au programme Coplamar (Coordinacion general del plan nacional de zonas pauperizadas y grupos marginados), développé à partir de 1976 à destination des populations rurales les plus pauvres (Bey, 2008). 
3. Área Geo Estadística Básica. Aire géo-statistique de base. Ces unités, instaurées par l'INEGI institut national de la statistique et de l'information géographique - à partir de 1990, délimitent une partie d'une localité de 2500 habitants ou plus, ou un chef-lieu municipal, indépendamment de sa population, en ensembles qui vont généralement de 1 à 50 îlots (manzanas).

4. Entre autres, le programme Progresa-Oportunidades a ainsi reposé sur la classification des localités selon l'indice de marginación établi par la CONAPO (Bey, 2008).

5. Pour une revue des différentes approches de la pauvreté, voir Destremau et Salama (2002).

6. La population visée par l'indice est précisément décrite comme celle «que por lo general se asienta de manera irregular en la periferia de las ciudades, en zonas no aptas para el desarrollo urbano (...)» (CONAPO, 2005, p. 11). Pour une approche critique, voir entre autres Gutiérrez-Pulido, Gama-Hernández (2010).

7. C'est suivant les contours de la zone métropolitaine de la vallée de Mexico (ZMVM) que la ville est appréhendée dans notre étude. En 2010, la ZMVM compte 76 unités administratives, réparties sur les 16 délégations du District Fédéral, 56 municipes de l'État de Mexico et 1 municipe de l'État d'Hidalgo (SEDESOL, CONAPO, INEGI, 2012). Dans la suite du texte, les termes de «ville », d'aire métropolitaine ou encore le toponyme « Mexico » renvoient systématiquement aux contours de la ZMVM.

8. On peut définir une approche substantialiste comme celle qui consiste à déterminer les caractéristiques sociales, économiques, démographiques susceptibles de témoigner des situations de pauvreté. C'est l'approche autorisée par les recensements de la population. La pauvreté peut bien entendu être cernée selon d'autres points de vue: une approche institutionnelle vise à cerner la pauvreté du point de vue de la relation spécifique qui se noue entre le pauvre et la société qui l'entoure; une approche subjective permet d'appréhender la pauvreté telle qu'elle ressort des expériences vécues (Ribardière et al., 2014).

9. Elles renvoient en particulier aux mesures de la pauvreté effectuées par le PNUD (Programme des Nations Unies pour le développement) : l'indice de pauvreté multidimensionnelle (IPM) repose sur la mesure de privations dans le domaine de la santé, de l'éducation et du niveau de vie (PNUD, 2010). Cette approche repose directement sur la notion de capabilité formulée par Amartya Sen (1987).

10. La dimension monétaire de la pauvreté n'a pas été retenue dans cette étude, en premier lieu parce que l'information n'est pas disponible à l'échelon fin de l'ageb. Des mesures de pauvreté intégrant la dimension monétaire existent au Mexique (Boltvinik, Hernández Laos, 1999) : elles sont effectuées par le CONEVAL (Consejo Nacional de Evaluación de la Política del Desarrollo Social) et reposent, pour les indicateurs de revenus, sur une enquête spécifique (ENIGH Encuesta Nacional de Ingresos y Gastos de los Hogares - 2006) dont les résultats sont diffusés à l'échelon des États et des localités urbaines uniquement. Il n'en reste pas moins que l'interprétation des revenus déclarés pose problème étant donné le poids du travail informel (Damián, 2008).

11. On peut se référer aux réflexions de D. Hiernaux-Nicolas sur les mécanismes de l'insertion urbaine des « indigènes ", notamment à Valle de Chalco (2005), et aux travaux de D. Delaunay (2007) sur le lien entre pauvreté et migration dans un contexte de discrimination ethnique au Mexique.

12. Selon un communiqué du CONEVAL de 2010, à l'occasion de la journée internationale des populations indigènes. Cf. également CONAPO $(2005,2012)$.

13. Après le cycle primaire - obligatoire, de 6 à 14 ans -, le cycle secondaire se découpe en deux temps : un premier stade d'éducation «basique » (3 ans) pour les 12-16 ans, un deuxième stade de 3 ans, le «bachillerato", sert de passerelle entre le secondaire et l'université ou le milieu professionnel. Primaire et première partie du secondaire font partie de ce qui est considéré comme «l'éducation basique ». Le deuxième stade du secondaire renvoie à un niveau de diplôme " post-basique ». Au-delà, les diplômes sont 
considérés comme "supérieurs ». Le recensement de la population de 2010 ne permet pas de distinguer, à l'échelon de l'ageb, le niveau "post-basique » du niveau « supérieur ».

14. Le Seguro Popular est un programme d'accès à la protection sociale et sanitaire destiné aux bas revenus, aux chômeurs ou aux travailleurs indépendants n'ayant pas accès à un régime de protection.

15. Moyenne à l'échelon des ageb pour la ZMVM, à celui des localités pour le Mexique.

16. À différencier de ceux que Blanchon (2010) appelle les "non branchés ", obligés de se ravitailler à des puits ou des bornes fontaines, ou par l'intermédiaire de systèmes alternatifs souvent onéreux comme les citernes. Ces «non-branchés » représentent encore une part non négligeable à l'échelle du Mexique, y compris dans les zones urbaines, même si Mexico occupe une position relativement privilégiée.

17. L'analyse des corrélations des variables prises deux à deux encourage cette démarche : les coefficients entre la part de la population « indigène », du point de vue de la langue, et les autres indicateurs, sont parfois faibles, mais toujours significatifs. Les coefficients témoignent d'une relation forte avec les indicateurs à la taille et à la suroccupation des logements (Valette, 2014b, p. 243).

18. La notion d'information est ici comprise au sens statistique, elle renvoie à la part de la variance totale du nuage de points expliquée par la position des unités géographiques sur un axe donné.

19. Cette partition résume $70 \%$ de l'information contenue dans les 4 premières composantes, soit $53,2 \%$ de l'information contenue dans le tableau de données initial.

20. Selon Duhau et Giglia (2008, p. 186), sur les zones urbanisées entre 1990 et 2000, 17,4\% sont des villages périurbains éloignés et $41,5 \%$ sont des grands ensembles d'accès aidé à la propriété formelle d'intérêt social.

21. La structure des covariations spatiales des indicateurs de pauvreté reste sensiblement la même lorsqu'elle est observée à l'échelle des seules 3430 ageb relevant de la catégorie des colonies populaires (les corrélations sont très fortes entre les coordonnées des axes de l'ACP globale et de l'ACP sur les colonies populaires : 0.99 entre les axes 1, 0.88 entre les axes 2, 0.80 entre les axes 3 et 0.82 entre les axes 4 ). D'un point de vue méthodologique, cette similarité des structures observées à l'échelle de la ville d'une part, et des colonies populaires d'autre part, autorise l'observation de la diversité des colonies populaires à travers la même grille de lecture que celle adoptée pour l'ensemble de la ville.

22. La thèse de consolidation/amélioration de l'installation illégale fut proposée par John Turner dans son rapport d'enquête (1966), Incontrolled Urban Settlement (Connolly, 2005).

23. Au Mexique, il existe trois types de propriété : sociale (la forme la plus répandue étant l'ejido au statut historiquement invendable et inaliénable), publique et privée. En 1992, les modifications de l'article 27 de la Constitution - garant de l'inaliénabilité des terres de propriété sociale - ont ouvert la voie d'une possible privatisation du foncier urbain, pourtant sous régime de propriété sociale, notamment dans la périphérie de la ville, au nord et à l'est. Mais son application au sein du DF est plus complexe, en raison des divers statuts de protection mis en place pour éviter l'étalement urbain. 


\section{ABSTRACTS}

In Mexico, urban poverty has been largely addressed through the concept of marginalidad theorized during the popular development of colonias populares, which were the main modality of physical growing of the city since the 1940s. This notion allows us to understand both social and spatial forms of poverty. Today, the link between poverty and popular colony needs to be revisited. From the 2010 population census, we offer an updated picture of the socio-spatial forms of poverty in Mexico City. Then, we examine the specific forms of settlement of colonias populares. The high diversity highlighted refers to paths consolidation and political dimension of the regularization process, typical of this mode of settlement.

Au Mexique, la pauvreté urbaine a été largement abordée à travers la notion de marginalidad, théorisée en écho au développement des colonies populaires, principale modalité d'expansion physique de la ville depuis les années 1940. Cette notion a ainsi permis de saisir conjointement les formes sociales et spatiales de la pauvreté. Aujourd'hui, le lien entre colonie populaire et pauvreté demande à être revisité. À partir du recensement de la population de 2010, nous proposons une image actualisée des formes socio-spatiales de la pauvreté dans la ville de Mexico, pour ensuite interroger la spécificité des formes de peuplement relevant des colonies populaires. La forte diversité mise en évidence renvoie aux trajectoires de consolidation et à la dimension politique du processus de régularisation, caractéristiques de ce mode de peuplement.

\section{INDEX}

Mots-clés: Mexico, quartier populaire, pauvreté urbaine, division sociale de l'espace Keywords: Mexico City, low income area, urban poverty, social division of space

\section{AUTHORS}

\section{ANTONINE RIBARDIÈRE}

Antonine Ribardière, antonine.ribardiere@univ-paris1.fr, est Maître de conférences à l'Université Paris 1 Panthéon-Sorbonne et membre de l'UMR 8586 PRODIG. Elle a publié récemment :

- Ribardière A., Bonerandi-Richard E., Martin M., Merchez L., 2014. La pauvreté dans l'espace européen : grilles de lecture. In Boulineau E., Bonerandi-Richard E. (dir.), La pauvreté en Europe, PUR, p. 17-32.

- Ribardière A., Valette J.-F., 2014. Évolution de la division sociale de l'espace urbain à Mexico. Approche à partir des structures démographiques et de l'accès au diplôme (1990-2010). Problèmes d'Amérique latine, $\mathrm{n}^{\circ}$ 90, p. 99-126.

\section{JEAN-FRANÇOIS VALETTE}

Jean-François Valette, jfrancois.valette@gmail.com, est docteur en géographie, Université Paris 1 Panthéon-Sorbonne et membre de l'UMR 8586 PRODIG. Il a publié récemment :

- Mesclier E., Piron M., Ribardiere A., Valette J-F., Lortic B., 2014. Divisions de l'espace résidentiel : une comparaison entre deux métropoles du Sud, Lima et Mexico. In Chaléard J.-L. (dir.), Métropoles aux Suds. Le défi des périphéries ?, Khartala, Prodig, Paris, Chapitre 6, p. 81-100. 
- Valette J-F., 2014. La régularisation territoriale des quartiers populaires de Mexico : une approche des rapports de pouvoir dans la maturation des périphéries. In Chaléard J.-L. (dir.), Métropoles aux Suds. Le défi des périphéries ?, Khartala, Prodig, Paris, Chapitre 24, p. 369-384. 